\title{
Erratum to: Evolutionary modularity and morphological integration in the haptoral anchor structures of Ligophorus spp. (Monogenea: Dactylogyridae)
}

\author{
A. Rodríguez-González ${ }^{1}$ - R. Míguez-Lozano ${ }^{1}$ - V. Sarabeev ${ }^{2}$ - J. A. Balbuena ${ }^{1}$
}

Published online: 24 November 2016

(C) Springer-Verlag Berlin Heidelberg 2016

\section{Erratum to: Parasitology Research 115(9): 3519-3533}

DOI 10.1007/s00436-016-5117-z

After online publication of this article, the authors recognized an error in the Materials and Methods section. The correct statement is shown below. The authors would like to point out that this error does not affect the interpretation of the results in the article.

"We based our morphological analysis on 286 individuals belonging to 14 valid species of Ligophorus for which original drawings of anchors were available: Ligophorus acuminatus Euzet \& Suriano, 1977; Ligophorus cephali Rubtsova et al., 2006; Ligophorus chabaudi Euzet \& Suriano, 1977;
Ligophorus confusus Euzet \& Suriano, 1977; Ligophorus heteronchus Euzet \& Suriano, 1977; Ligophorus imitans Euzet \& Suriano, 1977; Ligophorus macrocolpos Euzet \& Suriano 1977; Ligophorus mediterraneus Sarabeev et al., 2005; Ligophorus minimus Euzet \& Suriano, 1977; Ligophorus szidati Euzet \& Suriano, 1977; Ligophorus vanbenedenii Euzet \& Suriano, 1977; Ligophorus llewellyni; Ligophorus pilengas, and Ligophorus angustus Euzet \& Suriano, 1977. The sample size for each species was 20 individuals for ventral and 20 individuals for dorsal anchors (not always matching specimens of the previous group), except in L. angustus (four ventral and two dorsal anchors). In all, 526 anchors were studied of which, in 238 instances, represented ventral and dorsal anchors of the same worm individual".

The online version of the original article can be found at http://dx.doi. org/10.1007/s00436-016-5117-z.

A. Rodríguez-González

arogon5@uv.es

1 Marine Zoology Unit, Cavanilles Institute of Biodiversity and Evolutionary Biology, Science Park, University of Valencia, P.O. Box 22085, 46071 Valencia, Spain

2 Department of Biology, Zaporizhzhia National University, Zhukovskogo 66, 69063 Zhaporizhzhia, Ukraine 